\title{
A Hilbert-type integral inequality in the whole plane related to the kernel of exponent function
}

\author{
Yanru Zhong ${ }^{1 *}$, Meifa Huang ${ }^{2}$ and Bicheng Yang ${ }^{3}$
}

\section{"Correspondence:}

18577399236@163.com

'School of Computer Science and Information Security, Guilin

University of Electronic Technology, Guilin, P.R. China

Full list of author information is

available at the end of the article

\begin{abstract}
By using real analysis and weight functions, we obtain a few equivalent statements of a Hilbert-type integral inequality in the whole plane related to the kernel of exponent function with intermediate variables. The constant factor related to the gamma function is proved to be the best possible. We also consider some particular cases and the operator expressions.
\end{abstract}

MSC: 26D15; 31A10

Keywords: Hilbert-type integral inequality; Weight function; Intermediate variable; Equivalent statement; Operator; Gamma function

\section{Introduction}

If $0<\int_{0}^{\infty} f^{2}(x) d x<\infty$ and $0<\int_{0}^{\infty} g^{2}(y) d y<\infty$, then we have the following well-known Hilbert integral inequality (see [1]):

$$
\int_{0}^{\infty} \int_{0}^{\infty} \frac{f(x) g(y)}{x+y} d x d y<\pi\left(\int_{0}^{\infty} f^{2}(x) d x \int_{0}^{\infty} g^{2}(y) d y\right)^{\frac{1}{2}}
$$

where the constant factor $\pi$ is the best possible. In 1925, by introducing the pair of conjugate exponents $(p, q)\left(p>1, \frac{1}{p}+\frac{1}{q}=1\right)$, Hardy et al. gave an extension of (1) (see [1], Theorem 316). Recently, by means of weight functions, some new extensions of (1) and the Hardy's work were given by Yang [2, 3] and in [4-9]. Most of them are built in the quarter plane of the first quadrant.

In 2007, Yang [10] provided a Hilbert-type integral inequality in the whole plane with the exponent function and intermediate variables as follows:

$$
\int_{-\infty}^{\infty} \int_{-\infty}^{\infty} \frac{f(x) g(y)}{\left(1+e^{x+y}\right)^{\lambda}} d x d y<B\left(\frac{\lambda}{2}, \frac{\lambda}{2}\right)\left(\int_{-\infty}^{\infty} e^{-\lambda x} f^{2}(x) d x \int_{-\infty}^{\infty} e^{-\lambda y} g^{2}(y) d y\right)^{\frac{1}{2}}
$$

where the constant factor $B\left(\frac{\lambda}{2}, \frac{\lambda}{2}\right)$ is the best possible $(\lambda>0, B(u, v)$ is the beta function). He et al. [11-19] proved some new Hilbert-type integral inequalities in the whole plane with the best possible constant factors.

(c) The Author(s) 2018. This article is distributed under the terms of the Creative Commons Attribution 4.0 International License (http://creativecommons.org/licenses/by/4.0/), which permits unrestricted use, distribution, and reproduction in any medium, provided you give appropriate credit to the original author(s) and the source, provide a link to the Creative Commons license, and indicate if changes were made. 
In 2017, Hong [20] gave two equivalent statements between Hilbert-type inequalities with general homogenous kernel and a few parameters. A few authors continue to study this topic (see [21-25]).

In this paper, by using real analysis and weight functions we obtain a few equivalent statements of a Hilbert-type integral inequality in the whole plane related to the exponent function with intermediate variables. The constant factor related to the gamma function is proved to be the best possible. We also consider some particular cases and operator expressions.

\section{Some lemmas}

For $\gamma, \rho, \sigma>0$, setting $h(u):=e^{-\rho u^{\gamma}}(u>0)$, we find

$$
\begin{aligned}
k_{\rho}^{(\gamma)}(\sigma) & :=\int_{0}^{\infty} h(u) u^{\sigma-1} d u=\int_{0}^{\infty} e^{-\rho u^{\gamma}} u^{\sigma-1} d u \quad\left(v=\rho u^{\gamma}\right) \\
& =\frac{1}{\gamma \rho^{\sigma / \gamma}} \int_{0}^{\infty} e^{-v} v^{(\sigma / \gamma)-1} d v=\frac{\Gamma(\sigma / \gamma)}{\gamma \rho^{\sigma / \gamma}} \in \mathbb{R}_{+}=(0, \infty),
\end{aligned}
$$

where $\Gamma(s):=\int_{0}^{\infty} e^{-v} v^{s-1} d v(\operatorname{Re} s>0)$ is the gamma function (see [26]).

For $\delta \in\{-1,1\}, \alpha, \beta \in(-1,1)$, we set

$$
\begin{aligned}
& x_{\alpha}:=|x|+\alpha x, \quad y_{\beta}:=|y|+\beta y \quad(x, y \in \mathbb{R}=(-\infty, \infty)), \\
& E_{\delta}:=\left\{t \in \mathbb{R} ;|t|^{\delta} \geq 1\right\}, \quad E_{-\delta}=\left\{t \in \mathbb{R} ;|t|^{\delta} \leq 1\right\} .
\end{aligned}
$$

Lemma 1 For $c>0, \theta=\alpha, \beta \in(-1,1)$, we have

$$
\begin{aligned}
& \int_{E_{\delta}} t_{\theta}^{-c \delta-1} d t=\frac{1}{c}\left[\frac{1}{(1+\theta)^{c \delta+1}}+\frac{1}{(1-\theta)^{c \delta+1}}\right] \\
& \int_{E_{-\delta}} t_{\theta}^{c \delta-1} d t=\frac{1}{c}\left[\frac{1}{(1+\theta)^{-c \delta+1}}+\frac{1}{(1-\theta)^{-c \delta+1}}\right]
\end{aligned}
$$

and for $c \leq 0$, we have

$$
\int_{E_{\delta}} t_{\theta}^{-c \delta-1} d t=\int_{E_{-\delta}} t_{\theta}^{c \delta-1} d t=\infty
$$

Proof Setting $E_{\delta}^{+}:=\left\{t \in \mathbb{R}_{+} ; t^{\delta} \geq 1\right\}, E_{\delta}^{-}:=\left\{-t \in \mathbb{R}_{+} ;(-t)^{\delta} \geq 1\right\}$, we find $E_{\delta}=E_{\delta}^{+} \cup E_{\delta}^{-}$and

$$
\begin{aligned}
\int_{E_{\delta}} t_{\theta}^{-c \delta-1} d t & =\int_{E_{\delta}^{+}}[(1+\theta) t]^{-c \delta-1} d t+\int_{E_{\delta}^{-}}[(1-\theta)(-t)]^{-c \delta-1} d t \\
& =\left[\frac{1}{(1+\theta)^{c \delta+1}}+\frac{1}{(1-\theta)^{c \delta+1}}\right] \int_{E_{\delta}^{+}} t^{-c \delta-1} d t
\end{aligned}
$$

Setting $t=u^{\frac{1}{\delta}}$, we find

$$
\int_{E_{\delta}^{+}} t^{-c \delta-1} d t=\frac{1}{|\delta|} \int_{1}^{\infty} u^{\frac{1}{\delta}(-c \delta-1)} u^{\frac{1}{\delta}-1} d u=\int_{1}^{\infty} u^{-c-1} d u
$$


Hence, for $c>0$, (4) follows, and for $c \leq 0, \int_{E_{\delta}} t_{\theta}^{-c \delta-1} d t=\infty$. Since, for $c>0$,

$$
\int_{E_{-\delta}} t_{\theta}^{c \delta-1} d t=\int_{\left.E_{(-\delta}\right)} t_{\theta}^{-c(-\delta)-1} d t=\left[\frac{1}{(1+\theta)^{-c \delta+1}}+\frac{1}{(1-\theta)^{-c \delta+1}}\right] \int_{0}^{1} u^{c-1} d u
$$

we have (5), and for $c \leq 0, \int_{E_{-\delta}} t_{\theta}^{c \delta-1} d t=\infty$.

The lemma is proved.

In the following, We further assume that $p>1, \frac{1}{p}+\frac{1}{q}=1, \delta \in\{-1,1\}, \alpha, \beta \in(-1,1)$, $\gamma, \rho, \sigma>0, \sigma_{1} \in \mathbb{R}, k_{\rho}^{(\gamma)}(\sigma)$ is given by (3), and

$$
K_{\alpha, \beta}^{(\gamma)}(\sigma):=\frac{2 k_{\rho}^{(\gamma)}(\sigma)}{\left(1-\alpha^{2}\right)^{1 / q}\left(1-\beta^{2}\right)^{1 / p}}
$$

For $n \in \mathbb{N}=\{1,2, \ldots\}, E_{-1}=[-1,1], x \in E_{\delta}$, we define:

$$
\begin{aligned}
& I^{(-)}(x):=\int_{-1}^{0} e^{-\rho\left(x_{\alpha}^{\delta} y_{\beta}\right)^{\gamma}} y_{\beta}^{\sigma+\frac{1}{q^{n}}-1} d y, \quad I^{(+)}(x):=\int_{0}^{1} e^{-\rho\left(x_{\alpha}^{\delta} y_{\beta}\right)^{\gamma}} y_{\beta}^{\sigma+\frac{1}{q^{n}}-1} d y, \\
& I(x):=I^{(-)}(x)+I^{(+)}(x)=\int_{E_{-1}} e^{-\rho\left(x_{\alpha}^{\delta} y_{\beta}\right)^{\gamma}} y_{\beta}^{\sigma+\frac{1}{q^{n}}-1} d y .
\end{aligned}
$$

For $y_{\beta}=(\operatorname{sgn}(y)+\beta) y$, where

$$
\begin{aligned}
& \operatorname{sgn}(y):= \begin{cases}-1, & y<0, \\
0, & y=0, \\
1, & y>0,\end{cases} \\
& x_{\alpha}^{\delta}=(1+\alpha \operatorname{sgn}(x))^{\delta}|x|^{\delta} \geq \min _{\delta \in\{-1,1\}}\left\{(1 \pm|\alpha|)^{\delta}\right\} \quad\left(x \in E_{\delta}\right),
\end{aligned}
$$

and $1-|\alpha| \leq(1+|\alpha|)^{-1} \leq 1+|\alpha| \leq(1-|\alpha|)^{-1}$, we have

$$
(1 \pm \beta) x_{\alpha}^{\delta} \geq m_{\alpha, \beta}:=(1-|\beta|)(1-|\alpha|)>0 \quad\left(x \in E_{\delta}\right)
$$

For fixed $x \in E_{\delta}$, setting $u=x_{\alpha}^{\delta} y_{\beta}$, we find

$$
\begin{aligned}
& I^{(-)}(x)=\frac{x_{\alpha}^{-\delta\left(\sigma+\frac{1}{q n}\right)}}{1-\beta} \int_{0}^{(1-\beta) x_{\alpha}^{\delta}} e^{-\rho u^{\gamma}} u^{\sigma+\frac{1}{q^{n}}-1} d u \geq \frac{x_{\alpha}^{-\delta\left(\sigma+\frac{1}{q n}\right)}}{1-\beta} \int_{0}^{m_{\alpha, \beta}} e^{-\rho u^{\gamma}} u^{\sigma+\frac{1}{q^{n}}-1} d u, \\
& I^{(+)}(x)=\frac{x_{\alpha}^{-\delta\left(\sigma+\frac{1}{q n}\right)}}{1+\beta} \int_{0}^{(1+\beta) x_{\alpha}^{\delta}} e^{-\rho u^{\gamma}} u^{\sigma+\frac{1}{q n}-1} d u \geq \frac{x_{\alpha}^{-\delta\left(\sigma+\frac{1}{q n}\right)}}{1+\beta} \int_{0}^{m_{\alpha, \beta}} e^{-\rho u^{\gamma}} u^{\sigma+\frac{1}{q n}-1} d u, \\
& I(x)=x_{\alpha}^{-\delta\left(\sigma+\frac{1}{q n}\right)}\left[\frac{1}{1-\beta} \int_{0}^{(1-\beta) x_{\alpha}^{\delta}} e^{-\rho u^{\gamma}} u^{\sigma+\frac{1}{q n}-1} d u+\frac{1}{1+\beta} \int_{0}^{(1+\beta) x_{\alpha}^{\delta}} e^{-\rho u^{\gamma}} u^{\sigma+\frac{1}{q n}-1} d u\right] \\
& \geq \frac{2 x_{\alpha}^{-\delta\left(\sigma+\frac{1}{q n}\right)}}{1+\beta} \int_{0}^{m_{\alpha, \beta}} e^{-\rho u^{\gamma}} u^{\sigma+\frac{1}{q n}-1} d u .
\end{aligned}
$$


Zhong et al. Journal of Inequalities and Applications

(2018) 2018:234

Page 4 of 14

For $n \in \mathbb{N}=\{1,2, \ldots\}, x \in E_{-\delta}$, we define:

$$
\begin{aligned}
& J^{(-)}(x):=\int_{-\infty}^{-1} e^{-\rho\left(x_{\alpha}^{\delta} y_{\beta}\right)^{\gamma}} y_{\beta}^{\sigma-\frac{1}{q n}-1} d y, \\
& J^{(+)}(x):=\int_{1}^{\infty} e^{-\rho\left(x_{\alpha}^{\delta} y_{\beta}\right)^{\gamma}} y_{\beta}^{\sigma-\frac{1}{q n}-1} d y, \\
& J(x):=J^{(-)}(x)+J^{(+)}(x)=\int_{E_{-1}} e^{-\rho\left(x_{\alpha}^{\delta} y_{\beta}\right)^{\gamma}} y_{\beta}^{\sigma-\frac{1}{q^{n}}-1} d y .
\end{aligned}
$$

Since, for $x \in E_{-\delta}$,

$$
x_{\alpha}^{\delta}=(1+\alpha \operatorname{sgn}(x))^{\delta}|x|^{\delta} \leq \max _{\delta \in\{-1,1\}}\left\{(1 \pm|\alpha|)^{\delta}\right\}=(1-|\alpha|)^{-1},
$$

we have

$$
M_{\alpha, \beta}:=(1+|\beta|)(1-|\alpha|)^{-1} \geq(1 \pm|\beta|) x_{\alpha}^{\delta} \quad\left(x \in E_{-\delta}\right) .
$$

For fixed $x \in E_{-\delta}$, setting $u=x_{\alpha}^{\delta} y_{\beta}$, we find

$$
\begin{aligned}
& J^{(-)}(x)=\frac{x_{\alpha}^{-\delta\left(\sigma-\frac{1}{q n}\right)}}{1-\beta} \int_{(1-\beta) x_{\alpha}^{\delta}}^{\infty} e^{-\rho u^{\gamma}} u^{\sigma-\frac{1}{q n}-1} d u \geq \frac{x_{\alpha}^{-\delta\left(\sigma-\frac{1}{q n}\right)}}{1-\beta} \int_{M_{\alpha, \beta}}^{\infty} e^{-\rho u^{\gamma}} u^{\sigma-\frac{1}{q n}-1} d u, \\
& J^{(+)}(x)=\frac{x_{\alpha}^{-\delta\left(\sigma-\frac{1}{q n}\right)}}{1+\beta} \int_{(1+\beta) x_{\alpha}^{\delta}}^{\infty} e^{-\rho u^{\gamma}} u^{\sigma-\frac{1}{q^{n}}-1} d u \geq \frac{x_{\alpha}^{-\delta\left(\sigma-\frac{1}{q n}\right)}}{1+\beta} \int_{M_{\alpha, \beta}}^{\infty} e^{-\rho u^{\gamma}} u^{\sigma-\frac{1}{q^{n}}-1} d u, \\
& J(x)=x_{\alpha}^{-\delta\left(\sigma-\frac{1}{q n}\right)}\left[\frac{1}{1-\beta} \int_{(1-\beta) x_{\alpha}^{\delta}}^{\infty} e^{-\rho u^{\gamma}} u^{\sigma-\frac{1}{q^{n}}-1} d u+\frac{1}{1+\beta} \int_{(1+\beta) x_{\alpha}^{\delta}}^{\infty} e^{-\rho u^{\gamma}} u^{\sigma-\frac{1}{q n}-1} d u\right] \\
& \geq \frac{2 x_{\alpha}^{-\delta\left(\sigma-\frac{1}{q n}\right)}}{1+\beta} \int_{M_{\alpha, \beta}}^{\infty} e^{-\rho u^{\gamma}} u^{\sigma-\frac{1}{q n}-1} d u .
\end{aligned}
$$

In view of (8) and (10), we have the following:

Lemma 2 We have the following inequalities:

$$
\begin{aligned}
I_{1} & :=\int_{E_{\delta}} I(x) x_{\alpha}^{\delta\left(\sigma_{1}-\frac{1}{p n}\right)-1} d x \\
& \geq \frac{2}{1-\beta^{2}} \int_{E_{\delta}} x_{\alpha}^{-\delta\left(\sigma-\sigma_{1}+\frac{1}{n}\right)-1} d x \int_{0}^{m_{\alpha, \beta}} e^{-\rho u^{\gamma}} u^{\sigma+\frac{1}{q n}-1} d u \\
J_{1} & :=\int_{E_{-\delta}} J(x) x_{\alpha}^{\delta\left(\sigma_{1}+\frac{1}{p n}\right)-1} d x \\
& \geq \frac{2}{1-\beta^{2}} \int_{E_{-\delta}} x_{\alpha}^{\delta\left(\sigma_{1}-\sigma+\frac{1}{n}\right)-1} d x \int_{M_{\alpha, \beta}}^{\infty} e^{-\rho u^{\gamma}} u^{\sigma-\frac{1}{q n}-1} d u .
\end{aligned}
$$


Lemma 3 If there exists a constant $M$ such that, for any nonnegative measurable functions $f(x)$ and $g(y)$ in $\mathbb{R}$,

$$
\begin{aligned}
I & :=\int_{-\infty}^{\infty} \int_{-\infty}^{\infty} e^{-\rho\left(x_{\alpha}^{\delta} y_{\beta}\right)^{\gamma}} f(x) g(y) d x d y \\
& \leq M\left[\int_{-\infty}^{\infty} x_{\alpha}^{p\left(1-\delta \sigma_{1}\right)-1} f^{p}(x) d x\right]^{\frac{1}{p}}\left[\int_{-\infty}^{\infty} y_{\beta}^{q(1-\sigma)-1} g^{q}(y) d y\right]^{\frac{1}{q}}
\end{aligned}
$$

then we have $\sigma_{1}=\sigma$.

Proof If $\sigma_{1}>\sigma$, then for $n \geq \frac{1}{\sigma_{1}-\sigma}(n \in \mathbb{N})$, we define the functions:

$$
f_{n}(x):=\left\{\begin{array}{ll}
x_{\alpha}^{\delta\left(\sigma_{1}-\frac{1}{p n}\right)-1}, & x \in E_{\delta}, \\
0, & x \in \mathbb{R} \backslash E_{\delta},
\end{array} \quad g_{n}(y):= \begin{cases}y_{\beta}^{\sigma+\frac{1}{q^{n}}-1}, & y \in E_{-1}, \\
0, & y \in \mathbb{R} \backslash E_{-1},\end{cases}\right.
$$

and by (4) and (5) it follows that

$$
\begin{aligned}
\tilde{J}_{1} & :=\left[\int_{-\infty}^{\infty} x_{\alpha}^{p\left(1-\delta \sigma_{1}\right)-1} f_{n}^{p}(x) d x\right]^{\frac{1}{p}}\left[\int_{-\infty}^{\infty} y_{\beta}^{q(1-\sigma)-1} g_{n}^{q}(y) d y\right]^{\frac{1}{q}} \\
& =\left(\int_{E_{\delta}} x_{\alpha}^{-\frac{\delta}{n}-1} d x\right)^{\frac{1}{p}}\left(\int_{E_{-1}} y_{\beta}^{\frac{1}{n}-1} d y\right)^{\frac{1}{q}} \\
& =n\left[\frac{1}{(1+\alpha)^{\frac{\delta}{n}+1}}+\frac{1}{(1-\alpha)^{\frac{\delta}{n}+1}}\right]^{\frac{1}{p}}\left[\frac{1}{(1+\beta)^{\frac{-1}{n}+1}}+\frac{1}{(1-\beta)^{\frac{-1}{n}+1}}\right]^{\frac{1}{q}}<\infty .
\end{aligned}
$$

By (11) and (13) (for $f=f_{n}, g=g_{n}$ ) we have

$$
\begin{aligned}
& \frac{2}{1-\beta^{2}} \int_{E_{\delta}} x_{\alpha}^{-\delta\left(\sigma-\sigma_{1}+\frac{1}{n}\right)-1} d x \int_{0}^{m_{\alpha, \beta}} e^{-\rho u^{\gamma}} u^{\sigma+\frac{1}{q n}-1} d u \\
& \leq I_{1}=\int_{-\infty}^{\infty} \int_{-\infty}^{\infty} e^{-\rho\left(x_{\alpha}^{\delta} y_{\beta}\right)^{\gamma}} f_{n}(x) g_{n}(y) d x d y \leq M \tilde{J}_{1}<\infty
\end{aligned}
$$

Since for any $n \geq \frac{1}{\sigma_{1}-\sigma}, \sigma-\sigma_{1}+\frac{1}{n} \leq 0$, by Lemma 1 it follows that $\int_{E_{\delta}} x_{\alpha}^{-\delta\left(\sigma-\sigma_{1}+\frac{1}{n}\right)-1} d x=\infty$. In view of $\int_{0}^{m_{\alpha, \beta}} e^{-\rho u^{\gamma}} u^{\sigma+\frac{1}{q n}-1} d u>0$, we find that $\infty \leq M \tilde{J}_{1}<\infty$, which is a contradiction.

If $\sigma_{1}<\sigma$, then for $n \geq \frac{1}{\sigma-\sigma_{1}}(n \in \mathbb{N})$, we define the functions:

$$
\tilde{f}_{n}(x):=\left\{\begin{array}{ll}
x_{\alpha}^{\delta\left(\sigma+\frac{1}{p n}\right)-1}, & x \in E_{-\delta}, \\
0, & x \in \mathbb{R} \backslash E_{-\delta},
\end{array} \quad \tilde{g}_{n}(y):= \begin{cases}y_{\beta}^{\sigma-\frac{1}{q^{n}}-1}, & y \in E_{1}, \\
0, & y \in \mathbb{R} \backslash E_{1},\end{cases}\right.
$$


and by (4) and (5) it follows that

$$
\begin{aligned}
\tilde{J}_{2} & :=\left[\int_{-\infty}^{\infty} x_{\alpha}^{p\left(1-\delta \sigma_{1}\right)-1} \tilde{f}_{n}^{p}(x) d x\right]^{\frac{1}{p}}\left[\int_{-\infty}^{\infty} y_{\beta}^{q(1-\sigma)-1} \tilde{g}_{n}^{q}(y) d y\right]^{\frac{1}{q}} \\
& =\left(\int_{E_{-}} x_{\alpha}^{\frac{\delta}{n}-1} d x\right)^{\frac{1}{p}}\left(\int_{E_{1}} y_{\beta}^{\frac{-1}{n}-1} d y\right)^{\frac{1}{q}} \\
& =n\left[\frac{1}{(1+\alpha)^{\frac{-\delta}{n}+1}}+\frac{1}{(1-\alpha)^{\frac{-\delta}{n}}+1}\right]^{\frac{1}{p}}\left[\frac{1}{(1+\beta)^{\frac{1}{n}+1}}+\frac{1}{(1-\beta)^{\frac{\bar{n}}{n}+1}}\right]^{\frac{1}{q}}<\infty .
\end{aligned}
$$

By (12) and (13) (for $f=\tilde{f}_{n}, g=\tilde{g}_{n}$ ) we have

$$
\begin{aligned}
& \frac{2}{1-\beta^{2}} \int_{E_{-\delta}} x_{\alpha}^{\delta\left(\sigma_{1}-\sigma+\frac{1}{n}\right)-1} d x \int_{M_{\alpha, \beta}}^{\infty} e^{-\rho u^{\gamma}} u^{\sigma-\frac{1}{q^{n}}-1} d u \\
& \quad \leq J_{1}=\int_{-\infty}^{\infty} \int_{-\infty}^{\infty} e^{-\rho\left(x_{\alpha}^{\delta} y_{\beta}\right)^{\gamma}} \tilde{f}_{n}(x) \tilde{g}_{n}(y) d x d y \leq M \tilde{J}_{2}<\infty
\end{aligned}
$$

Since for any $n \geq \frac{1}{\sigma-\sigma_{1}}, \sigma_{1}-\sigma+\frac{1}{n} \leq 0$, by Lemma 1 it follows that $\int_{E_{-\delta}} x_{\alpha}^{\delta\left(\sigma_{1}-\sigma+\frac{1}{n}\right)-1} d x=$ $\infty$. In view of $\int_{M_{\alpha, \beta}}^{\infty} e^{-\rho u^{\gamma}} u^{\sigma-\frac{1}{q n}-1} d u>0$, we have $\infty \leq M \tilde{J}_{2}<\infty$, which is a contradiction.

Hence we conclude that $\sigma_{1}=\sigma$.

The lemma is proved.

Lemma 4 If there exists a constant $M$ such that, for any nonnegative measurable functions $f(x)$ and $g(y)$ in $\mathbb{R}$,

$$
\begin{aligned}
& \int_{-\infty}^{\infty} \int_{-\infty}^{\infty} e^{-\rho\left(x_{\alpha}^{\delta} y_{\beta}\right)^{\gamma}} f(x) g(y) d x d y \\
& \quad \leq M\left[\int_{-\infty}^{\infty} x_{\alpha}^{p(1-\delta \sigma)-1} f^{p}(x) d x\right]^{\frac{1}{p}}\left[\int_{-\infty}^{\infty} y_{\beta}^{q(1-\sigma)-1} g^{q}(y) d y\right]^{\frac{1}{q}},
\end{aligned}
$$

then we have $K_{\alpha, \beta}^{(\gamma)}(\sigma) \leq M$.

Proof For $\sigma_{1}=\sigma$, by (8) we have

$$
\begin{aligned}
& I_{1}=\int_{E_{\delta}} I(x) x_{\alpha}^{\delta\left(\sigma-\frac{1}{p n}\right)-1} d x=I_{1}^{(-)}+I_{1}^{(+)} \\
& I_{1}^{(-)}=\int_{E_{\delta}} I^{(-)}(x) x_{\alpha}^{\delta\left(\sigma-\frac{1}{p n}\right)-1} d x, \quad I_{1}^{(+)}:=\int_{E_{\delta}} I^{(+)}(x) x_{\alpha}^{\delta\left(\sigma-\frac{1}{p n}\right)-1} d x
\end{aligned}
$$

In view of the presented results, we find

$$
\begin{aligned}
I_{1}^{(-)} & =\frac{1}{1-\beta} \int_{E_{\delta}} x_{\alpha}^{-\frac{\delta}{n}-1} \int_{0}^{(1-\beta) x_{\alpha}^{\delta}} e^{-\rho u^{\gamma}} u^{\sigma+\frac{1}{q n}-1} d u d x \\
& =\frac{1}{1-\beta} \int_{E_{\delta}} x_{\alpha}^{-\frac{\delta}{n}-1}\left[\int_{0}^{\infty} e^{-\rho u^{\gamma}} u^{\sigma+\frac{1}{q n}-1} d u-\int_{(1-\beta) x_{\alpha}^{\delta}}^{\infty} e^{-\rho u^{\gamma}} u^{\sigma+\frac{1}{q n}-1} d u\right] d x
\end{aligned}
$$




$$
\begin{aligned}
= & \frac{n}{1-\beta}\left[\frac{1}{(1+\alpha)^{\frac{\delta}{n}+1}}+\frac{1}{(1-\alpha)^{\frac{\delta}{n}+1}}\right] k_{\rho}^{(\gamma)}\left(\sigma+\frac{1}{q n}\right) \\
& -\frac{1}{1-\beta} \int_{E_{\delta}} x_{\alpha}^{-\frac{\delta}{n}-1} \int_{(1-\beta) x_{\alpha}^{\delta}}^{\infty} e^{-\rho u^{\gamma}} u^{\sigma+\frac{1}{q n}-1} d u d x .
\end{aligned}
$$

Since $e^{-\rho u^{\gamma}} u^{2 \sigma}$ is continuous in $(0, \infty)$, and $e^{-\rho u^{\gamma}} u^{2 \sigma} \rightarrow 0(u \rightarrow \infty)$, there exists a positive constant $M_{1}$ such that $e^{-\rho u^{\gamma}} u^{2 \sigma} \leq M_{1}\left(u \in\left[m_{\alpha, \beta}, \infty\right)\right)$. By (4) it follows that

$$
\begin{aligned}
0 & <\int_{E_{\delta}} x_{\alpha}^{-\frac{\delta}{n}-1} \int_{(1-\beta) x_{\alpha}^{\delta}}^{\infty} e^{-\rho u^{\gamma}} u^{\sigma+\frac{1}{q n}-1} d u d x \\
& \leq M_{1} \int_{E_{\delta}} x_{\alpha}^{-\frac{\delta}{n}-1}\left[\int_{(1-\beta) x_{\alpha}^{\delta}}^{\infty} u^{-\sigma+\frac{1}{q n}-1} d u\right] d x=\frac{M_{1} \int_{E_{\delta}} x_{\alpha}^{-\delta\left(\sigma+\frac{1}{p n}\right)-1} d x}{(1-\beta)^{\sigma-\frac{1}{q n}}} \\
& =\frac{\left(\sigma+\frac{1}{p n}\right)^{-1} M_{1}}{(1-\beta)^{\sigma-\frac{1}{q n}}}\left[\frac{1}{(1+\alpha)^{\delta\left(\sigma+\frac{1}{p n}\right)+1}}+\frac{1}{(1-\alpha)^{\delta\left(\sigma+\frac{1}{p n}\right)+1}}\right]
\end{aligned}
$$

So that

$$
\frac{1}{1-\beta} \int_{E_{\delta}} x_{\alpha}^{-\frac{\delta}{n}-1} \int_{(1-\beta) x_{\alpha}^{\delta}}^{\infty} e^{-\rho u^{\gamma}} u^{\sigma+\frac{1}{q n}-1} d u d x=O(1)
$$

By (15) it follows that

$$
\frac{1}{n} I_{1}^{(-)}=\frac{k_{\rho}^{(\gamma)}\left(\sigma+\frac{1}{q n}\right)}{1-\beta}\left[\frac{1}{(1+\alpha)^{\frac{\delta}{n}+1}}+\frac{1}{(1-\alpha)^{\frac{\delta}{n}+1}}\right]-\frac{O(1)}{n} .
$$

In the same way, we have

$$
\frac{1}{n} I_{1}^{(+)}=\frac{k_{\rho}^{(\gamma)}\left(\sigma+\frac{1}{q n}\right)}{1+\beta}\left[\frac{1}{(1+\alpha)^{\frac{\delta}{n}+1}}+\frac{1}{(1-\alpha)^{\frac{\delta}{n}+1}}\right]-\frac{\tilde{O}(1)}{n} .
$$

By (14) (for $\left.f=f_{n}, g=g_{n}\right)$, we have

$$
\frac{1}{n} I_{1}=\frac{1}{n}\left(I_{1}^{(-)}+I_{1}^{(+)}\right) \leq \frac{1}{n} M \tilde{J}_{1}
$$

For $n \rightarrow \infty$, by Fatou lemma (see [27]), (16), and (17) we find

$$
\frac{2}{1-\beta^{2}} \frac{2 k_{\rho}^{(\gamma)}(\sigma)}{1-\alpha^{2}} \leq M\left(\frac{2}{1-\alpha^{2}}\right)^{\frac{1}{p}}\left(\frac{2}{1-\beta^{2}}\right)^{\frac{1}{q}}
$$

so that $K_{\alpha, \beta}^{(\gamma)}(\sigma)=\frac{2 k_{\rho}^{(\gamma)}(\sigma)}{\left(1-\alpha^{2}\right)^{1 / q}\left(1-\beta^{2}\right)^{1 / p}} \leq M$

The lemma is proved.

Lemma 5 We define the following weight functions:

$$
\omega_{\delta}(\sigma, y):=y_{\beta}^{\sigma} \int_{-\infty}^{\infty} e^{-\rho\left(x_{\alpha}^{\delta} y_{\beta}\right)^{\gamma}} x_{\alpha}^{\delta \sigma-1} d x \quad(y \in \mathbb{R})
$$




$$
\varpi_{\delta}(\sigma, x):=x_{\alpha}^{\delta \sigma} \int_{-\infty}^{\infty} e^{-\rho\left(x_{\alpha}^{\delta} y_{\beta}\right)^{\gamma}} y_{\beta}^{\sigma-1} d y \quad(x \in \mathbb{R})
$$

Then we have

$$
\frac{1-\alpha^{2}}{2} \omega_{\delta}(\sigma, y)=\frac{1-\beta^{2}}{2} \varpi_{\delta}(\sigma, x)=k_{\rho}^{(\gamma)}(\sigma) \quad(x, y \in \mathbb{R} \backslash\{0\})
$$

Proof For fixed $y \in \mathbb{R} \backslash\{0\}$, setting $u=x_{\alpha}^{\delta} y_{\beta}$, we find

$$
\begin{aligned}
\omega_{\delta}(\sigma, y)= & y_{\beta}^{\sigma} \int_{-\infty}^{0} e^{-\rho\left(x_{\alpha}^{\delta} y_{\beta}\right)^{\gamma}}[(1-\alpha)(-x)]^{\delta \sigma-1} d x \\
& +y_{\beta}^{\sigma} \int_{0}^{\infty} e^{-\rho\left(x_{\alpha}^{\delta} y_{\beta}\right)^{\gamma}}[(1+\alpha) x]^{\delta \sigma-1} d x \\
= & \left(\frac{1}{1-\alpha}+\frac{1}{1+\alpha}\right) \int_{0}^{\infty} e^{-\rho u^{\gamma}} u^{\sigma-1} d u=\frac{2}{1-\alpha^{2}} k_{\rho}^{(\gamma)}(\sigma) ;
\end{aligned}
$$

for fixed $x \in \mathbb{R} \backslash\{0\}$, setting $u=x_{\alpha}^{\delta} y_{\beta}$, it follows that

$$
\begin{aligned}
\varpi_{\delta}(\sigma, x) & =x_{\alpha}^{\delta \sigma} \int_{-\infty}^{0} e^{-\rho\left(x_{\alpha}^{\delta} y_{\beta}\right)^{\gamma}} y_{\beta}^{\sigma-1} d y+x_{\alpha}^{\delta \sigma} \int_{0}^{\infty} e^{-\rho\left(x_{\alpha}^{\delta} y_{\beta}\right)^{\gamma}} y_{\beta}^{\sigma-1} d y \\
& =\frac{2}{1-\beta^{2}} \int_{0}^{\infty} e^{-\rho u^{\gamma}} u^{\sigma-1} d u=\frac{2}{1-\beta^{2}} k_{\rho}^{(\gamma)}(\sigma) .
\end{aligned}
$$

Hence we have (20).

The lemma is proved.

\section{Main results}

Theorem 1 If $M$ is a constant, then the following statements (i), (ii), and (iii) are equivalent:

(i) For any $f(x) \geq 0$, we have:

$$
\begin{aligned}
J & :=\left\{\int_{-\infty}^{\infty} y_{\beta}^{p \sigma-1}\left[\int_{-\infty}^{\infty} e^{-\rho\left(x_{\alpha}^{\delta} y_{\beta}\right)^{\gamma}} f(x) d x\right]^{p} d y\right\}^{\frac{1}{p}} \\
& \leq M\left[\int_{-\infty}^{\infty} x_{\alpha}^{p\left(1-\delta \sigma_{1}\right)-1} f^{p}(x) d x\right]^{\frac{1}{p}} .
\end{aligned}
$$

(ii) For any $f(x), g(y) \geq 0$, we have:

$$
\begin{aligned}
I & =\int_{-\infty}^{\infty} \int_{-\infty}^{\infty} e^{-\rho\left(x_{\alpha}^{\delta} y_{\beta}\right)^{\gamma}} f(x) g(y) d x d y \\
& \leq M\left[\int_{-\infty}^{\infty} x_{\alpha}^{p\left(1-\delta \sigma_{1}\right)-1} f^{p}(x) d x\right]^{\frac{1}{p}}\left[\int_{-\infty}^{\infty} y_{\beta}^{q(1-\sigma)-1} g^{q}(y) d y\right]^{\frac{1}{q}} .
\end{aligned}
$$

(iii) $\sigma_{1}=\sigma$, and $K_{\alpha, \beta}^{(\gamma)}(\sigma) \leq M$ 
Proof (i) $=>$ (ii). By Hölder's inequality (see [28]) we have

$$
\begin{aligned}
I & =\int_{-\infty}^{\infty}\left[y_{\beta}^{\sigma-\frac{1}{p}} \int_{-\infty}^{\infty} e^{-\rho\left(x_{\alpha}^{\delta} y_{\beta}\right)^{\gamma}} f(x) d x\right]\left(y_{\beta}^{-\sigma+\frac{1}{p}} g(y)\right) d y \\
& \leq J\left[\int_{-\infty}^{\infty} y_{\beta}^{q(1-\sigma)-1} g^{q}(y) d y\right]^{\frac{1}{q}} .
\end{aligned}
$$

Then by (21) we have (22).

(ii) $=>$ (iii). By Lemma 1 we have $\sigma_{1}=\sigma$. Then by Lemma 2 we have $K_{\alpha, \beta}^{(\gamma)}(\sigma) \leq M$.

(iii) $=>$ (i). For $\sigma_{1}=\sigma$, by Hölder's inequality with weight (see [28]) and (18) we have

$$
\begin{aligned}
& {\left[\int_{-\infty}^{\infty} e^{-\rho\left(x_{\alpha}^{\delta} y_{\beta}\right)^{\gamma}} f(x) d x\right]^{p}} \\
& \quad=\left\{\int_{-\infty}^{\infty} e^{-\rho\left(x_{\alpha}^{\delta} y_{\beta}\right)^{\gamma}}\left[\frac{y_{\beta}^{(\sigma-1) / p} f(x)}{x_{\alpha}^{(\delta \sigma-1) / q}}\right]\left[\frac{x_{\alpha}^{(\delta \sigma-1) / q}}{y_{\beta}^{(\sigma-1) / p}}\right] d x\right\}^{p} \\
& \quad \leq \int_{-\infty}^{\infty} e^{-\rho\left(x_{\alpha}^{\delta} y_{\beta}\right)^{\gamma}} \frac{y_{\beta}^{\sigma-1} f^{p}(x)}{x_{\alpha}^{(\delta \sigma-1) p / q}} d x\left[\int_{-\infty}^{\infty} e^{-\rho\left(x_{\alpha}^{\delta} y_{\beta}\right)^{\gamma}} \frac{x_{\alpha}^{\delta \sigma-1}}{y_{\beta}^{(\sigma-1) q / p}} d x\right]^{p / q} \\
& =\left[\omega_{\delta}(\sigma, y) y_{\beta}^{q(1-\sigma)-1}\right]^{p-1} \int_{-\infty}^{\infty} e^{-\rho\left(x_{\alpha}^{\delta} y_{\beta}\right)^{\gamma}} \frac{y_{\beta}^{\sigma-1} f^{p}(x)}{x_{\alpha}^{(\delta \sigma-1) p / q}} d x \\
& =\left(\frac{2 k_{\rho}^{(\gamma)}(\sigma)}{1-\alpha^{2}}\right)^{p-1} y_{\beta}^{-p \sigma+1} \int_{-\infty}^{\infty} e^{-\rho\left(x_{\alpha}^{\delta} y_{\beta}\right)^{\gamma}} \frac{y_{\beta}^{\sigma-1} f^{p}(x)}{x_{\alpha}^{(\delta \sigma-1) p / q}} d x .
\end{aligned}
$$

By Fubini's theorem (see [27]), (24), and (19) we have

$$
\begin{aligned}
J & \leq\left(\frac{2 k_{\rho}^{(\gamma)}(\sigma)}{1-\alpha^{2}}\right)^{\frac{1}{q}}\left[\int_{-\infty}^{\infty} \int_{-\infty}^{\infty} e^{-\rho\left(x_{\alpha}^{\delta} y_{\beta}\right)^{\gamma}} \frac{y_{\beta}^{\sigma-1} f^{p}(x)}{x_{\alpha}^{(\delta \sigma-1) p / q}} d x d y\right]^{\frac{1}{p}} \\
& =\left(\frac{2 k_{\rho}^{(\gamma)}(\sigma)}{1-\alpha^{2}}\right)^{\frac{1}{q}}\left[\int_{-\infty}^{\infty} \varpi_{\delta}(\sigma, x) x_{\delta}^{p(1-\delta \sigma)-1} f^{p}(x) d x\right]^{\frac{1}{p}} \\
& =K_{\alpha, \beta}^{(\gamma)}(\sigma)\left[\int_{-\infty}^{\infty} x_{\delta}^{p(1-\delta \sigma)-1} f^{p}(x) d x\right]^{\frac{1}{p}} .
\end{aligned}
$$

For $K_{\alpha, \beta}^{(\gamma)}(\sigma) \leq M$, we have (21) (when $\sigma_{1}=\sigma$ ).

Therefore, statements (i), (ii), and (iii) are equivalent.

The theorem is proved.

Theorem 2 If $M$ is a constant, then the following statements (i), (ii), and (iii) are equivalent:

(i) For any $f(x) \geq 0$ satisfying $0<\int_{-\infty}^{\infty} x_{\alpha}^{p(1-\delta \sigma)-1} f^{p}(x) d x<\infty$, we have:

$$
\begin{aligned}
& \left\{\int_{-\infty}^{\infty} y_{\beta}^{p \sigma-1}\left[\int_{-\infty}^{\infty} e^{-\rho\left(x_{\alpha}^{\delta} y_{\beta}\right)^{\gamma}} f(x) d x\right]^{p} d y\right\}^{\frac{1}{p}} \\
& <M\left[\int_{-\infty}^{\infty} x_{\alpha}^{p(1-\delta \sigma)-1} f^{p}(x) d x\right]^{\frac{1}{p}} .
\end{aligned}
$$


(ii) For any $f(x) \geq 0$ satisfying $0<\int_{-\infty}^{\infty} x_{\alpha}^{p(1-\delta \sigma)-1} f^{p}(x) d x<\infty$, and $g(x) \geq 0$ satisfying $0<\int_{-\infty}^{\infty} y_{\beta}^{q(1-\sigma)-1} g^{q}(y) d y<\infty$, we have:

$$
\begin{aligned}
& \int_{-\infty}^{\infty} \int_{-\infty}^{\infty} e^{-\rho\left(x_{\alpha}^{\delta} y_{\beta}\right)^{\gamma}} f(x) g(y) d x d y \\
& \quad<M\left[\int_{-\infty}^{\infty} x_{\alpha}^{p(1-\delta \sigma)-1} f^{p}(x) d x\right]^{\frac{1}{p}}\left[\int_{-\infty}^{\infty} y_{\beta}^{q(1-\sigma)-1} g^{q}(y) d y\right]^{\frac{1}{q}} .
\end{aligned}
$$

(iii) $K_{\alpha, \beta}^{(\gamma)}(\sigma) \leq M$

Moreover, if statement (iii) holds, then the constant factor $M=K_{\alpha, \beta}^{(\gamma)}(\sigma)$ in (25) and (26) is the best possible.

In particular, (1) for $\delta=1, M=K_{\alpha, \beta}^{(\gamma)}(\sigma)$, we have the following equivalent inequalities with nonhomogeneous kernel:

$$
\begin{aligned}
& \left\{\int_{-\infty}^{\infty} y_{\beta}^{p \sigma-1}\left[\int_{-\infty}^{\infty} e^{-\rho\left(x_{\alpha} y_{\beta}\right)^{\gamma}} f(x) d x\right]^{p} d y\right\}^{\frac{1}{p}} \\
& <K_{\alpha, \beta}^{(\gamma)}(\sigma)\left[\int_{-\infty}^{\infty} x_{\alpha}^{p(1-\sigma)-1} f^{p}(x) d x\right]^{\frac{1}{p}}, \\
& \int_{-\infty}^{\infty} \int_{-\infty}^{\infty} e^{-\rho\left(x_{\alpha} y_{\beta}\right)^{\gamma}} f(x) g(y) d x d y \\
& \quad<K_{\alpha, \beta}^{(\gamma)}(\sigma)\left[\int_{-\infty}^{\infty} x_{\alpha}^{p(1-\sigma)-1} f^{p}(x) d x\right]^{\frac{1}{p}}\left[\int_{-\infty}^{\infty} y_{\beta}^{q(1-\sigma)-1} g^{q}(y) d y\right]^{\frac{1}{q}}
\end{aligned}
$$

where $K_{\alpha, \beta}^{(\gamma)}(\sigma)$ is the best possible constant factor;

(2) for $\delta=-1, M=K_{\alpha, \beta}^{(\gamma)}(\sigma)$, we have the following equivalent inequalities with homogeneous kernel of degree 0 :

$$
\begin{aligned}
& \left\{\int_{-\infty}^{\infty} y_{\beta}^{p \sigma-1}\left[\int_{-\infty}^{\infty} e^{-\rho\left(y_{\beta} / x_{\alpha}\right)^{\gamma}} f(x) d x\right]^{p} d y\right\}^{\frac{1}{p}} \\
& <K_{\alpha, \beta}^{(\gamma)}(\sigma)\left[\int_{-\infty}^{\infty} x_{\alpha}^{p(1+\sigma)-1} f^{p}(x) d x\right]^{\frac{1}{p}}, \\
& \int_{-\infty}^{\infty} \int_{-\infty}^{\infty} e^{-\rho\left(y_{\beta} / x_{\alpha}\right)^{\gamma}} f(x) g(y) d x d y \\
& \quad<K_{\alpha, \beta}^{(\gamma)}(\sigma)\left[\int_{-\infty}^{\infty} x_{\alpha}^{p(1+\sigma)-1} f^{p}(x) d x\right]^{\frac{1}{p}}\left[\int_{-\infty}^{\infty} y_{\beta}^{q(1-\sigma)-1} g^{q}(y) d y\right]^{\frac{1}{q}}
\end{aligned}
$$

where $K_{\alpha, \beta}^{(\gamma)}(\sigma)$ is the best possible constant factor.

Proof For $\sigma_{1}=\sigma$, under and the assumption of statement (i), if (24) takes the form of equality for $y \in \mathbb{R} \backslash\{0\}$, then there exist constants $A$ and $B$ such that they are not both zero and (see [28])

$$
A \frac{y_{\beta}^{\sigma-1}}{x_{\alpha}^{(\delta \sigma-1) p / q}} f^{p}(x)=B \frac{x_{\alpha}^{\delta \sigma-1}}{y_{\beta}^{(\sigma-1) q / p}} \quad \text { a.e. in } \mathbb{R} \text {. }
$$


We suppose that $A \neq 0$ (otherwise, $B=A=0$ ). Then it follows that

$$
x_{\alpha}^{p(1-\delta \sigma)-1} f^{p}(x)=y_{\beta}^{q(\sigma-1)} \frac{B}{A x_{\alpha}} \quad \text { a.e. in } \mathbb{R} .
$$

Since $\int_{-\infty}^{\infty} x_{\alpha}^{-1} d x=\infty$, this contradicts the fact that $0<\int_{-\infty}^{\infty} x_{\alpha}^{p(1-\delta \sigma)-1} f^{p}(x) d x<\infty$. Hence (24) takes the form of strict inequality, and so does (21). Hence (25) and (26) are valid.

In view of Theorem 1, we still can conclude that statements (i), (ii), and (iii) in Theorem 2 are equivalent.

When statement (iii) holds, namely, $K_{\alpha, \beta}^{(\gamma)}(\sigma) \leq M$, if there exists a constant $M\left(\leq K_{\alpha, \beta}^{(\gamma)}(\sigma)\right)$ such that (26) is valid, then $M=K_{\alpha, \beta}^{(\gamma)}(\sigma)$, and we can conclude that the constant factor $M=K_{\alpha, \beta}^{(\gamma)}(\sigma)$ in (26) is the best possible.

The constant factor $M=K_{\alpha, \beta}^{(\gamma)}(\sigma)$ in (25) is still the best possible. Otherwise, by (23) (for $\sigma_{1}=\sigma$ ), we would get a contradiction that the constant factor $M=K_{\alpha, \beta}^{(\gamma)}(\sigma)$ in (26) is not the best possible.

The theorem is proved.

\section{Operator expressions}

We set the following functions: $\varphi(x):=x_{\alpha}^{p(1-\delta \sigma)-1}(x \in \mathbb{R})$, and $\psi(y):=y_{\beta}^{q(1-\sigma)-1}$, where from $\psi^{1-p}(y):=y_{\beta}^{p \sigma-1}(y \in \mathbb{R})$. Define the following real normed linear spaces:

$$
\begin{aligned}
& L_{p, \varphi}(\mathbb{R}):=\left\{f ;\|f\|_{p, \varphi}:=\left(\int_{-\infty}^{\infty} \varphi(x)|f(x)|^{p} d x\right)^{\frac{1}{p}}<\infty\right\}, \\
& L_{q, \psi}(\mathbb{R}):=\left\{g ;\|g\|_{q, \psi}=\left(\int_{-\infty}^{\infty} \psi(y)|g(y)|^{q} d y\right)^{\frac{1}{q}}<\infty\right\}, \\
& L_{p, \psi^{1-p}}(\mathbb{R}):=\left\{h ;\|h\|_{p, \psi^{1-p}}=\left(\int_{-\infty}^{\infty} \psi^{1-p}(y)|h(y)|^{p} d y\right)^{\frac{1}{p}}<\infty\right\} .
\end{aligned}
$$

In view of Theorem 2 , for $f \in L_{p, \varphi}(\mathbb{R})$, setting

$$
h_{1}(y):=\int_{-\infty}^{\infty} e^{-\rho\left(y_{\beta} / x_{\alpha}\right)^{\gamma}} f(x) d x \quad(y \in \mathbb{R})
$$

by (25) we have

$$
\left\|h_{1}\right\|_{p, \psi^{1-p}}=\left(\int_{-\infty}^{\infty} \psi^{1-p}(y)\left|h_{1}(y)\right|^{p} d y\right)^{\frac{1}{p}} \leq M\|f\|_{p, \varphi}<\infty
$$

Definition 1 Define the Hilbert-type integral operator $T: L_{p, \varphi}(\mathbb{R}) \rightarrow L_{p, \psi^{1-p}}(\mathbb{R})$ as follows: For any $f \in L_{p, \varphi}(\mathbb{R})$, there exists a unique representation $T f=h_{1} \in L_{p, \psi^{1-p}}(\mathbb{R})$, satisfying for any $y \in \mathbb{R}, T f(y)=h_{1}(y)$.

In view of (31), it follows that $\|T f\|_{p, \psi^{1-p}}=\left\|h_{1}\right\|_{p, \psi^{1-p}} \leq M\|f\|_{p, \varphi}$, and then the operator $T$ is bounded and satisfies

$$
\|T\|=\sup _{f(\not \theta) \in L_{p, \varphi}(\mathbb{R})} \frac{\|T f\|_{p, \psi} 1-p}{\|f\|_{p, \varphi}} \leq M .
$$


If we define the formal inner product of $T f$ and $g$ as

$$
(T f, g):=\int_{-\infty}^{\infty}\left[\int_{-\infty}^{\infty} e^{-\rho\left(y_{\beta} / x_{\alpha}\right)^{\gamma}} f(x) d x\right] g(y) d y,
$$

then we can rewrite Theorem 2 as follows.

Theorem 3 If $M$ is a constant, then the following statements (i), (ii), and (iii) are equivalent:

(i) For any $f(x) \geq 0, f \in L_{p, \varphi}(\mathbb{R}),\|f\|_{p, \varphi}>0$, we have:

$$
\|T f\|_{p, \psi} 1-p<M\|f\|_{p, \varphi}
$$

(ii) $\operatorname{For} f(x), g(y) \geq 0, f \in L_{p, \varphi}(\mathbb{R}), g \in L_{q, \psi}(\mathbb{R}),\|f\|_{p, \varphi},\|g\|_{q, \psi}>0$, we have:

$$
(T f, g)<M\|f\|_{p, \varphi}\|g\|_{q, \psi} .
$$

(iii) $K_{\alpha, \beta}^{(\gamma)}(\sigma) \leq M$.

Moreover, if statement (iii) holds, then the constant factor $M=K_{\alpha, \beta}^{(\gamma)}(\sigma)$ in (32) and (33) is the best possible, namely, $\|T\|=K_{\alpha, \beta}^{(\gamma)}(\sigma)$.

Remark 1 (1) In particular, for $\alpha=\beta=0$ in (27) and (28), we have the following equivalent inequalities:

$$
\begin{aligned}
& \left\{\int_{-\infty}^{\infty}|y|^{p \sigma-1}\left[\int_{-\infty}^{\infty} e^{-\rho|x y|^{\gamma}} f(x) d x\right]^{p} d y\right\}^{\frac{1}{p}} \\
& <\frac{2 \Gamma(\sigma / \gamma)}{\gamma \rho^{\sigma / \gamma}}\left[\int_{-\infty}^{\infty}|x|^{p(1-\sigma)-1} f^{p}(x) d x\right]^{\frac{1}{p}} \\
& \int_{-\infty}^{\infty} \int_{-\infty}^{\infty} e^{-\rho|x y|^{\gamma}} f(x) g(y) d x d y \\
& <\frac{2 \Gamma(\sigma / \gamma)}{\gamma \rho^{\sigma / \gamma}}\left[\int_{-\infty}^{\infty}|x|^{p(1-\sigma)-1} f^{p}(x) d x\right]^{\frac{1}{p}}\left[\int_{-\infty}^{\infty}|y|^{q(1-\sigma)-1} g^{q}(y) d y\right]^{\frac{1}{q}},
\end{aligned}
$$

where $\frac{2 \Gamma(\sigma / \gamma)}{\gamma \rho^{\sigma / \gamma}}$ is the best possible constant factor.

If $f(-x)=f(x), g(-y)=g(y)\left(x, y \in \mathbb{R}_{+}\right)$, then we have the following equivalent inequalities:

$$
\begin{aligned}
& \left\{\int_{0}^{\infty} y^{p \sigma-1}\left[\int_{0}^{\infty} e^{-\rho(x y)^{\gamma}} f(x) d x\right]^{p} d y\right\}^{\frac{1}{p}} \\
& <\frac{\Gamma(\sigma / \gamma)}{\gamma \rho^{\sigma / \gamma}}\left[\int_{0}^{\infty} x^{p(1-\sigma)-1} f^{p}(x) d x\right]^{\frac{1}{p}} \\
& \int_{0}^{\infty} \int_{0}^{\infty} e^{-\rho(x y)^{\gamma}} f(x) g(y) d x d y \\
& <\frac{\Gamma(\sigma / \gamma)}{\gamma \rho^{\sigma / \gamma}}\left[\int_{0}^{\infty} x^{p(1-\sigma)-1} f^{p}(x) d x\right]^{\frac{1}{p}}\left[\int_{0}^{\infty} y^{q(1-\sigma)-1} g^{q}(y) d y\right]^{\frac{1}{q}}
\end{aligned}
$$

where $\frac{\Gamma(\sigma / \gamma)}{\gamma \rho^{\sigma / \gamma}}$ is the best possible constant factor. 
(2) For $\alpha=\beta=0$ in (29) and (30), we have the following equivalent inequalities:

$$
\begin{aligned}
& \left\{\int_{-\infty}^{\infty}|y|^{p \sigma-1}\left[\int_{-\infty}^{\infty} e^{-\rho|y / x|^{\gamma}} f(x) d x\right]^{p} d y\right\}^{\frac{1}{p}} \\
& <\frac{2 \Gamma(\sigma / \gamma)}{\gamma \rho^{\sigma / \gamma}}\left[\int_{-\infty}^{\infty}|x|^{p(1+\sigma)-1} f^{p}(x) d x\right]^{\frac{1}{p}} \\
& \int_{-\infty}^{\infty} \int_{-\infty}^{\infty} e^{-\rho|y / x|^{\gamma}} f(x) g(y) d x d y \\
& <\frac{2 \Gamma(\sigma / \gamma)}{\gamma \rho^{\sigma / \gamma}}\left[\int_{-\infty}^{\infty}|x|^{p(1+\sigma)-1} f^{p}(x) d x\right]^{\frac{1}{p}}\left[\int_{-\infty}^{\infty}|y|^{q(1-\sigma)-1} g^{q}(y) d y\right]^{\frac{1}{q}},
\end{aligned}
$$

where $\frac{2 \Gamma(\sigma / \gamma)}{\gamma \rho^{\sigma / \gamma}}$ is the best possible constant factor.

If $f(-x)=f(x), g(-y)=g(y)\left(x, y \in \mathbb{R}_{+}\right)$, then we have the following equivalent inequalities:

$$
\begin{aligned}
& \left\{\int_{0}^{\infty} y^{p \sigma-1}\left[\int_{0}^{\infty} e^{-\rho(y / x)^{\gamma}} f(x) d x\right]^{p} d y\right\}^{\frac{1}{p}} \\
& <\frac{\Gamma(\sigma / \gamma)}{\gamma \rho^{\sigma / \gamma}}\left[\int_{0}^{\infty} x^{p(1+\sigma)-1} f^{p}(x) d x\right]^{\frac{1}{p}} \\
& \int_{0}^{\infty} \int_{0}^{\infty} e^{-\rho(y / x)^{\gamma}} f(x) g(y) d x d y \\
& <\frac{\Gamma(\sigma / \gamma)}{\gamma \rho^{\sigma / \gamma}}\left[\int_{0}^{\infty} x^{p(1+\sigma)-1} f^{p}(x) d x\right]^{\frac{1}{p}}\left[\int_{0}^{\infty} y^{q(1-\sigma)-1} g^{q}(y) d y\right]^{\frac{1}{q}}
\end{aligned}
$$

where $\frac{\Gamma(\sigma / \gamma)}{\gamma \rho^{\sigma / \gamma}}$ is the best possible constant factor.

\section{Conclusions}

In this paper, by using real analysis and weight functions we obtain a few equivalent statements of a Hilbert-type integral inequality in the whole plane related to the kernel of exponent function with the intermediate variables (Theorem 1 ). The constant factor related to the gamma function is proved to be the best possible in Theorem 2. We also consider some particular cases and the operator expressions in Remark 1 and Theorem 3. The lemmas and theorems provide an extensive account of this type of inequalities.

\section{Funding}

This work is supported by the National Natural Science Foundation (Nos. 61562016 and 51765012) and Science and Technology Planning Project Item of Guangzhou City (No. 201707010229). We are grateful for this help.

\section{Competing interests}

The authors declare that they have no competing interests.

\section{Authors' contributions}

BY carried out the mathematical studies, participated in the sequence alignment, and drafted the manuscript. YZ and MH participated in the design of the study and performed the numerical analysis. All authors read and approved the final manuscript.

\section{Author details}

'School of Computer Science and Information Security, Guilin University of Electronic Technology, Guilin, P.R. China.

${ }^{2}$ School of Mechanical Science and Electrical Engineering, Guilin University of Electronic Technology, Guilin, P.R. China.

${ }^{3}$ Department of Mathematics, Guangdong University of Education, Guangzhou, P.R. China. 


\section{Publisher's Note}

Springer Nature remains neutral with regard to jurisdictional claims in published maps and institutional affiliations.

Received: 14 July 2018 Accepted: 2 September 2018 Published online: 12 September 2018

\section{References}

1. Hardy, G.H., Littlewood, J.E., Polya, G.: Inequalities. Cambridge University Press, Cambridge (1934)

2. Yang, B.C.: The Norm of Operator and Hilbert-Type Inequalities. Science Press, Beijing (2009)

3. Yang, B.C.: Hilbert-Type Integral Inequalities. Bentham Science Publishers, The United Arab Emirates (2009)

4. Yang, B.C.: On the norm of an integral operator and applications. J. Math. Anal. Appl. 321, 182-192 (2006)

5. Xu, J.S.: Hardy-Hilbert's inequalities with two parameters. Adv. Math. 36(2), 189-198 (2007)

6. Yang, B.C.: On the norm of a Hilbert's type linear operator and applications. J. Math. Anal. Appl. 325, 529-541 (2007)

7. Xin, D.M.: A Hilbert-type integral inequality with the homogeneous kernel of zero degree. Math. Theory Appl. 30(2), 70-74 (2010)

8. Yang, B.C.: A Hilbert-type integral inequality with the homogenous kernel of degree 0. J. Shandong Univ. Nat. Sci. 45(2), 103-106 (2010)

9. Debnath, L., Yang, B.C.: Recent developments of Hilbert-type discrete and integral inequalities with applications. Int. J. Math. Math. Sci. 2012, Article ID 871845 (2012)

10. Yang, B.C.: A new Hilbert-type integral inequality. Soochow J. Math. 33(4), 849-859 (2007)

11. He, B., Yang, B.C.: On a Hilbert-type integral inequality with the homogeneous kernel of 0-degree and the hypergeometric function. Math. Pract. Theory 40(18), 105-211 (2010)

12. Yang, B.C.: A new Hilbert-type integral inequality with some parameters. J. Jilin Univ. Sci. Ed. 46(6), 1085-1090 (2008)

13. Wang, A.Z., Yang, B.C.: A new Hilbert-type integral inequality in whole plane with the non-homogeneous kernel. J. Inequal. Appl. 2011, 123 (2011)

14. He, B., Yang, B.C.: On an inequality concerning a non-homogeneous kernel and the hypergeometric function. Tamsui Oxf. J. Inf. Math. Sci. 27(1), 75-88 (2011)

15. Xie, Z.T., Zeng, Z., Sun, Y.F.: A new Hilbert-type inequality with the homogeneous kernel of degree -2. Adv. Appl. Math. Sci. 12(7), 391-401 (2013)

16. Huang, Q.L., Wu, S.H., Yang, B.C.: Parameterized Hilbert-type integral inequalities in the whole plane. Sci. World J. 2014, Article ID 169061 (2014)

17. Zhen, Z., Raja Rama Gandhi, K., Xie, Z.T.: A new Hilbert-type inequality with the homogeneous kernel of degree -2 and with the integral. Bull. Math. Sci. Appl. 3(1), 11-20 (2014)

18. Rassias, M.Th., Yang, B.C.: A Hilbert-type integral inequality in the whole plane related to the hyper geometric function and the beta function. J. Math. Anal. Appl. 428(2), 1286-1308 (2015)

19. Gu, Z.H., Yang, B.C.: A Hilbert-type integral inequality in the whole plane with a non-homogeneous kernel and a few parameters. J. Inequal. Appl. 2015, 314 (2015)

20. Hong, Y:: On the structure character of Hilbert's type integral inequality with homogeneous kernel and applications. J. Jilin Univ. Sci. Ed. 55(2), 189-194 (2017)

21. Rassias, M.Th., Yang, B.C.: Equivalent properties of a Hilbert-type integral inequality with the best constant factor related the Hurwitz zeta function. Ann. Funct. Anal. 9(2), 282-295 (2018)

22. Hong, Y., Huang, Q.L., Yang, B.C., Liao, J.Q.: The necessary and sufficient conditions for the existence of a kind of Hilbert-type multiple integral inequality with the non-homogeneous kernel and its applications. J. Inequal. Appl. 2017, $316(2017)$

23. Yang, B.C., Chen, Q.: Equivalent conditions of existence of a class of reverse Hardy-type integral inequalities with nonhomogeneous kernel. J. Jilin Univ. Sci. Ed. 55(4), 804-808 (2017)

24. Yang, B.C.: Equivalent conditions of the existence of Hardy-type and Yang-Hilbert-type integral inequalities with the nonhomogeneous kernel. J. Guangdong Univ. Educ. 37(3), 5-10 (2017)

25. Yang, B.C.: On some equivalent conditions related to the bounded property of Yang-Hilbert-type operator. J. Guangdong Univ. Educ. 37(5), 5-11 (2017)

26. Wang, Z.Q., Guo, D.R.: Introduction to Special Functions. Science Press, Beijing (1979)

27. Kuang, J.C.: Real and Functional Analysis (Continuation) (Second Volume). Higher Education Press, Beijing (2015)

28. Kuang, J.C.: Applied Inequalities. Shangdong Science and Technology Press, Jinan (2004) $\varnothing$

\section{Submit your manuscript to a SpringerOpen ${ }^{\circ}$ journal and benefit from:}

- Convenient online submission

- Rigorous peer review

- Open access: articles freely available online

- High visibility within the field

- Retaining the copyright to your article

Submit your next manuscript at $\downarrow$ springeropen.com 